\title{
Rhinosinusitis in Secondary School Children-Part 2: Main project analysis of MSNOT-20 Young Persons Questionnaire $(M S Y P Q)^{*}$
}

\author{
Amtul S. Sami and Glenis K. Scadding \\ ENT and Allergy Department, Royal National Throat, Nose and Ear Hospital, London, United Kingdom
}

Rhinology 52: 225-230, 2014

DOI:10.4193/Rhino12.011-2

*Received for publication:

January 16, 2012

Accepted: December 28, 2013

\begin{abstract}
Background: There is little data on rhinosinusitis in adolescent schoolchildren. We have employed a validated, disease specific quality of life questionnaire to determine the extent of this problem and its effects on their lives.

Methododology: The MSYPQ, a disease specific quality of life questionnaire, previously evaluated in a pilot study of adolescent sino-nasal disease, was used in secondary school children in East London to identify the prevalence of rhinosinusitis plus its effects on quality of life. One group of the secondary school children completed ARIA based questions for comparison with MSYPQ rhinitis sub-scores.
\end{abstract}

Results: $71 \%$ scored an abnormal value on the MSYPQ for at least one symptom, 32\% of those assessed suffered from symptoms compatible with rhinitis, similar to the prevalence of $30 \%$ previously found in adults. Unlike adults cough was one of the most significant symptoms. Over $21 \%$ of secondary school students had their quality of life affected and $11 \%$ took time off school due to their symptoms. The ARIA assessment group showed that symptoms were intermittent in $44 \%$ and confirmed significant impairment of sleep and daily activities.

Conclusion: The MSYPQ demonstrates a high prevalence and impact on quality of life of rhinitis and rhinosinusitis symptoms in the 11-16 age group, with levels comparable to the results from an adult population. The MSYPQ rhinitis sub group of questions was concordant with ARIA based questions.

Key words: rhinitis, sinusitis, prevalence, EPOS, secondary school

\section{Introduction}

Rhinitis, when associated with inflammation of the paranasal sinuses, is known as rhinosinusitis and both occur frequently in the population ${ }^{(1,2)}$. There is a paucity of data on the extent of this problem in secondary schoolchildren; the main aims of this study were to identify the prevalence of rhinitis/rhinosinusitis in secondary school children and its effect on quality of life, secondary aims were to determine the degree of impairment due to these symptoms. To this end, while assessing the prevalence of nasal and paranasal symptoms within the age group of 11-16 years, the prevalence of impaired sleep, social and emotional function, time off school and visits to the family doctor were also evaluated.

To meet these aims, we used a modified SNOT-20 (MSNOT-20) questionnaire, which had already been validated and used in a large scale adult community based survey, the results of which identified the MSNOT-20 as a good instrument to explore the prevalence of rhinitis/rhinosinusitis and its effect on quality of life ${ }^{(3)}$. The MSNOT-20 questionnaire was modified according to 
the requirements of the age group being assessed and the suggestions of the ethical committee to make it appropriate to use in an interview-based and postal survey in older children. These modifications, discussed in Part 1, created the modified SNOT-20 for young person's questionnaire (MSYPQ) which consisted of three sections: demographic details, disease specific questions and quality of life/treatment questions. Following the pilot project (Part 1) the MSYPQ was identified as a valid, disease specific quality of life tool for the assessment of rhinitis/rhinosinusitis within the 11-16 year age group.

A copy of the MSYPQ is available in the Appendix of part 1.

The MSYPQ was used in children aged between 11-16 years from three major secondary schools in East London following informed consent by each head teacher, each child and their parents or carers.

The schools chosen were three of the largest secondary schools within the Newham borough, situated so that each school draws students from different areas of the borough. These schools: Kingsford Community (mixed), Brampton Manor (mixed) and Plashet school (girls only), collectively represent over 4,000 children aged 11-16 years living in the locality and represent the ethnic diversity of the area. In Brampton Manor and Plashet schools, a series of face-to-face interviews of the consenting student with the research doctor took place as well as postal surveys, while at Kingsford Community School, a postal survey only was carried out, due to clashes with examination timing and staffing issues.

\section{Materials and methods}

\section{Questionaire}

Following consent of the head teachers, the students were given a presentation and the opportunity to ask any questions before being given the information and consent form packs to take home, with a view to be a part of the research project following completion of the consent form.

In a face-to-face interview, under the 'quality of life' section, 70 students were also asked ARIA guideline based questions on symptom persistence, which were later analysed in accordance with results from the MSYPQ.

\section{Results}

\section{Response rates}

Two hundred and thirteen secondary school children (19.2\% male) took part. The interview based survey responses were 47.33\% (in Plashet school) and 20\% (in Brampton Manor school) while the postal response (at Kingsford Community school) was $11 \%$.
During data analysis, statistical testing showed that there was no significant difference between the male and female responders, nor between interview and postal questionnaires.

\section{Reliability Analysis}

The reliability test of the MSYPQ for this main project was carried out using Cronbach's alpha, a measure of internal consistency or reliability. There is a consensus that an alpha value of greater than 0.7 shows good reliability within an instrument (4). The reliability check showed the value of Cronbach's a was 0.990 , signifying that the instrument being used is reliable and is able to detect rhinosinusitis.

\section{Response rates}

The scores obtained are shown in Table 1.

Table 1. Mean and standard deviation for each question in disease specific section of the MSYPQ $(n=213)$.

\begin{tabular}{|c|c|c|}
\hline MSYPQ Question & Mean & $\begin{array}{l}\text { Standard } \\
\text { deviation }\end{array}$ \\
\hline Blocked nose & 1.76 & 1.706 \\
\hline Need to blow the nose & 1.5 & 1.560 \\
\hline Runny nose & 1.5 & 1.583 \\
\hline Sneezing & 1.46 & 1.484 \\
\hline Cough & 1.3 & 1.381 \\
\hline Wake up tired & 1.2 & 1.474 \\
\hline Lack of good night sleep & 0.95 & 1.425 \\
\hline Wake up at night & 0.9 & 1.361 \\
\hline Post nasal discharge & 0.83 & 1.263 \\
\hline Thick nasal discharge & 0.83 & 1.271 \\
\hline Difficulty falling a sleep & 0.77 & 1.232 \\
\hline Fatigue & 0.68 & 1.167 \\
\hline Ear fullness & 0.65 & 1.273 \\
\hline Reduce productivity & 0.65 & 1.100 \\
\hline Reduce concentration & 0.64 & 1.131 \\
\hline Frustrated restless/irritable & 0.64 & 1.131 \\
\hline Dizziness & 0.56 & 1.034 \\
\hline Ear pain & 0.52 & 1.062 \\
\hline Facial pain & 0.52 & 1.012 \\
\hline Embarrassed & 0.48 & 1.705 \\
\hline
\end{tabular}


The analysis of each score depends on knowledge of the cut -off value where a result ceases to be within the normal limits. This is identified by referring to the healthy group (EPOS negative) from the pilot project (Part 1) which provides the benchmark for normality.

Scores above 0.364 (mean plus 2 standard deviations above the healthy group in our pilot survey in which the overall mean was 0.09 while the standard deviation was 0.137 ) were attributed as abnormal. In total, $70.87 \%$ of responders had an abnormal score for any one of the sinus and/or nasal symptoms. Of these, $27.15 \%$ had their symptoms for less than six weeks, $31.13 \%$ had their symptoms for more than six weeks but for less than a year, $33.77 \%$ between one to five years and $7.95 \%$ had this problem for more than five years.

Table 1 shows the highest mean score is for blocked nose with a value of 1.76 , runny nose and need to blow the nose are next with a mean of 1.5 , while sneezing is 1.46 . The standard deviation of each score is significantly high, suggesting wide variation in the data.

At least $31.5 \%$ of the secondary school children had an abnormal score (a severity score of over 2 for each question, see part 1) on what we have termed the Rhinitic Index (a combination of the four individual nasal symptoms: blocked nose, need to blow the nose, sneezing and runny nose) indicating that at the time of the survey nearly $1 / 3$ of the secondary school children were suffering from rhinitis. A similar value of $30 \%$ was found in a comparable adult community based survey ${ }^{(3)}$.

Rhinitic symptoms were more prevalent than sinus-related problems, with the reporting of post nasal discharge and/or facial pressure/pain and/or thick nasal discharge being found in 13 $15 \%$ of secondary school children. Ear symptoms of fullness, ear pain and dizziness occurred in $15.1 \%, 11.3 \%$ and $7.1 \%$, respectively. Over $20 \%$ reported difficulty falling asleep, $15.5 \%$ complained of waking up at night and more than $17 \%$ noted lack of a good night's sleep. More than $20 \%$ of students woke up tired which led to reduced productivity in nearly $8 \%$ and reduced concentration in $12 \%$ of the students; over $13 \%$ suffered from fatigue and nearly $10 \%$ were frustrated due to these symptoms. This figure is similar to that in a research paper surveying GCSE results and hay fever ${ }^{(5)}$. In some cases the rhinitic symptoms were so severe that, as reported by the MSYPQ, just over $11 \%$ have had to take time off school in the past year.

There was a correlation of symptoms with family history of atopy: those who had a positive family history of asthma (found in $44.6 \%$ ), eczema (in 38\%), hay fever (80\%) and food allergy (44\%) tended to have higher MSYPQ scores, this was statistically

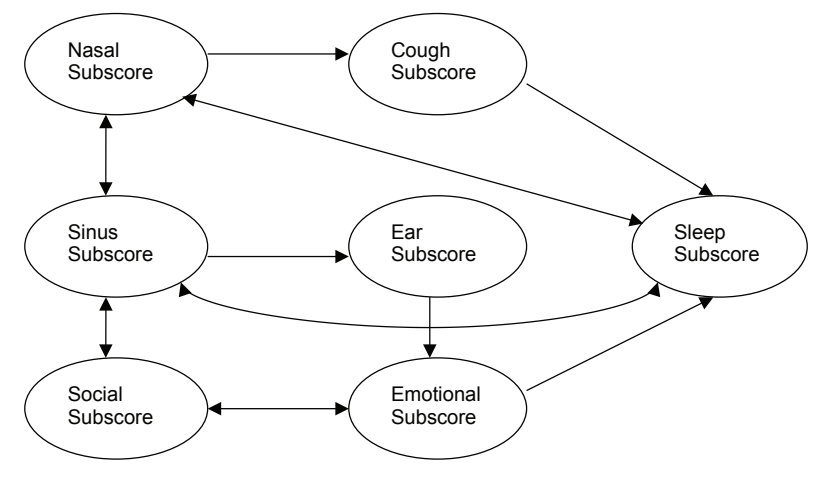

Figure 1. Correlation between individual subgroups using MSYPQ in association with Kendal tau b test table. The questions associated with each subgroup are as follows: Nasal: 1, 2, 3, 19; Paranasal: 5, 6, 7, 8, 9, 10; Sinus: 5, 6, 10; Ear: 7, 8, 9; Sleep: 11, 12, 13,14; Social: 15, 16, 17; Emotional: 18, 20.

significant, $\mathrm{p}<0.01$.

\section{Subgroup analysis}

The collection of questions regarding components of the same domain were combined together, on clinical grounds, to create subgroups which were then further analysed ${ }^{(3)}$. For example nasal symptoms were gathered from questions 1, 2, 3 and 19, paranasal from $5,6,7,8,9,10$ (further breakdown of sub groups can be found in Legend of Figure 1).

Table 2 shows the correlations between symptoms in a Kendal tau-b table. There were highly significant correlations within each sub group; this is diagrammatically represented in Figure 1.

One of the major correlations seen is that of the nasal sub group with sinus, sleep and social subgroups and with the individual question of cough. The strongest correlations were between nasal and paranasal scores and sleep, social and emotional scores.

In the MSYPQ there is a high score for cough which is related to nasal and sleep sub scores.

\section{ARIA questions analysis}

Analysis of the 70 ARIA questionnaires in self-reported nasal and sinus symptom sufferers showed that $44.28 \%$ of symptomatic students suffered intermittent rhinitis symptoms i.e. less than 4 days per week or less than 4 week at a time, whereas $51.42 \%$ suffered persistent symptoms i.e. more than 4 days per week and more than 4 weeks at a time. Due to nasal symptoms, $51.42 \%$ complained of abnormal sleep, $38.57 \%$ of impairment of daily activities, $37.14 \%$ of problems in their classroom activities and the same percentage of students complained of troublesome 
Table 2. Kendal tau b test table quantifying the correlation between individual questions of the MSYPQ.

\begin{tabular}{|c|c|c|c|c|c|c|c|c|c|c|c|c|c|c|c|c|c|c|c|c|}
\hline & Q1 & Q2 & Q3 & Q4 & Q5 & Q6 & Q7 & Q8 & Q9 & Q10 & Q11 & Q12 & Q13 & Q14 & Q15 & Q16 & Q17 & Q18 & Q19 & Q20 \\
\hline Need To Blow Nose Q1 & 1 & & & & & & & & & & & & & & & & & & & \\
\hline Sneezing Q2 & .983 & 1 & & & & & & & & & & & & & & & & & & \\
\hline Runny Nose Q3 & .966 & .954 & 1 & & & & & & & & & & & & & & & & & \\
\hline Cough Q4 & .928 & .927 & .967 & 1 & & & & & & & & & & & & & & & & \\
\hline Post Nasal Discharge Q5 & .872 & .838 & .885 & .894 & 1 & & & & & & & & & & & & & & & \\
\hline Thick Nasal Discharge Q6 & .855 & .828 & .878 & .878 & .946 & 1 & & & & & & & & & & & & & & \\
\hline Ear Fullness Q7 & .761 & .733 & .783 & .781 & .868 & .880 & 1 & & & & & & & & & & & & & \\
\hline Dizziness Q8 & .792 & .757 & .812 & .810 & .907 & .863 & .926 & 1 & & & & & & & & & & & & \\
\hline Ear Pain Q9 & .717 & .717 & .754 & .754 & .881 & .846 & .906 & .928 & 1 & & & & & & & & & & & \\
\hline Facial Pain Q10 & .739 & .739 & .807 & .814 & .885 & .848 & .816 & .881 & .841 & 1 & & & & & & & & & & \\
\hline Difficulty Falling Sleep Q11 & .853 & .833 & .858 & .844 & .873 & .914 & .861 & .860 & .794 & .815 & 1 & & & & & & & & & \\
\hline Wake Up Night Q12 & .858 & .833 & .910 & .894 & .899 & .881 & .851 & .900 & .844 & .910 & .920 & 1 & & & & & & & & \\
\hline $\begin{array}{l}\text { Lack Of Good Night Sleep } \\
\text { Q13 }\end{array}$ & .883 & .861 & .900 & .883 & .926 & .922 & .905 & .938 & .884 & .840 & .910 & .938 & 1 & & & & & & & \\
\hline Wake Up Tired Q14 & .895 & .886 & .945 & .946 & .901 & .895 & .831 & .871 & .822 & .827 & .868 & .922 & .937 & 1 & & & & & & \\
\hline Fatigue Q15 & .831 & .796 & .844 & .832 & .888 & .887 & .873 & .935 & .889 & .847 & .906 & .924 & .943 & .883 & 1 & & & & & \\
\hline Reduce Productivity Q16 & .873 & .813 & .846 & .835 & .858 & .902 & .858 & .867 & .805 & .837 & .947 & .919 & .910 & .859 & .945 & 1 & & & & \\
\hline Reduce Concentration Q17 & .848 & .821 & .878 & .873 & .921 & .923 & .870 & .919 & .883 & .848 & .894 & .927 & .963 & .926 & .957 & .924 & 1 & & & \\
\hline Frustrated Q18 & .793 & .778 & .800 & .785 & .816 & .872 & .888 & .850 & .808 & .730 & .854 & .831 & .902 & .839 & .887 & .892 & .902 & 1 & & \\
\hline Blocked Nose Q19 & .952 & .995 & .939 & .923 & .813 & .801 & .678 & .731 & .623 & .712 & .833 & .837 & .826 & .881 & .776 & .798 & .806 & .728 & 1 & \\
\hline Embarrassed Q20 & .748 & .693 & .745 & .736 & .876 & .820 & .849 & .918 & .885 & .888 & .853 & .873 & .869 & .784 & .895 & .859 & .868 & .775 & .680 & 1 \\
\hline
\end{tabular}

symptoms. Analysis also found that $41.63 \%$ have taken 1 to 5 days off school while $9.35 \%$ have taken 6 to 15 days off.

\section{Treatment}

$46.94 \%$ of young persons received treatment while $26.76 \%$ never took any treatment, the remaining (26.3\%) had not reported symptom warranting treatment. Of those treated, $45.07 \%$ used that prescribed after seeking medical advice, $13.61 \%$ obtained their treatment from the pharmacy whereas $7.51 \%$ used alternative medicine. $39.43 \%$ of the subjects did not bother to take advice from their GP in the past year. Of the $46.47 \%$ subjects who, at some point, took advice from GP about their nasal and sinus problems; $17.84 \%$ visited once, $23.47 \%$ twice, $6.57 \%$ three times, $5.16 \%$ of the subjects visited four times and $0.47 \%$ visited their GP 10 times about their symptoms. $4.22 \%$ of the subjects had severe symptoms and had been seen by specialist; $2.34 \%$ needed an operation.

For $55.86 \%$ of the subject's treatment worked; in $15.96 \%$ the treatment had no effect on their symptoms. $17.84 \%$ of the subjects used treatment on most days of the problem period, $10.79 \%$ used treatment twice a day while $10.32 \%$ used it once or twice per week.

91.54\% of the subjects suggested that their parents buy the medicine for them and had no idea about the cost

\section{Discussion}

Previous surveys have evaluated the prevalence of rhinitis/ rhinosinusitis in the community, but most involves adults, with 
little data at secondary school level. These research projects have, primarily, involved postal questionnaires or face to face interviews. The validity of the results from the latter is likely to be greater, as the questioner can check that the index case fully understands the question, but the former allows a much larger population sample and, as such, may give a more representative finding for the community. A postal survey within the UK in 1991 reported the prevalence of all form of rhinitis (excluding infectious) as $24 \%$ : $30 \%$ seasonal, $13 \%$ perennial and $57 \%$ mixed (6).

Rhinitis has been shown to significantly affect social well-being, school and learning performance ${ }^{(7-10)}$ as well as to impact on productivity and thus have a major impact on quality of life. In addition, the financial costs may be substantial ${ }^{(11)}$. A health care expenditure study in the USA found that children were responsible for $30.6 \%$ of the overall spending from government funding for their respiratory problems including sinusitis, otitis media, asthma and upper respiratory tract infections ${ }^{(12)}$.

Schipper et al. have defined HRQL (health related quality of life) as "the functional effects of an illness and its consequent therapy upon a patient, as perceived by the patient" ${ }^{\prime(13-15)}$. The MSYPQ was derived from the MSNOT-20 disease specific and health related quality of life tool which was developed following one modification of the well-validated SNOT-20 questionnaire ${ }^{(16-19)}$. This modification was done by changing the symptom of "sad" to "blocked nose" in order to capture a major problematic symptom, further modifications included adding a demographic section and quality of life questions as a final section.

Having validated the questionnaire for rhinosinusitis in a pilot study, the MSYPQ has been used to explore the prevalence of nasal and sinus symptoms in 11-16 year old secondary school children. The preponderance of females taking part may have skewed the results; however statistical analysis has shown there was no statistically significant difference between males and females during the data evaluation in our study.

The results from this study show that $70.87 \%$ in total have upper respiratory tract symptoms: $31.5 \%$ suffer from a combination of symptoms suggestive of rhinitis, more than $12 \%$ suffer from sinus symptoms and more than $20 \%$ of students suffered from effects on quality of life. These results are similar to a study where seasonal allergic rhinoconjunctivitis is estimated to affect $15-23 \%$ of children with peak age of onset in adolescence ${ }^{(5)}$.

In children the symptoms of rhinosinusitis are often difficult to distinguish from those of a common cold and allergic rhinitis. The most common acute rhinosinusitis symptoms include cough $80 \%$, nasal discharge $75 \%$ and malodorous breath $50 \%$
(12). As the MSYPQ asks about symptoms in the last two weeks, in addition to long lasting and persistent symptoms, the questionnaire will detect transient and short lived symptoms, such as those related to the common cold which is probably why so many were affected in our study which was carried out in winter. Seasonal influences would alter the response at different times of the year however this project was done outside of the hay fever season in an attempt to minimise these. In total, out of those with nasal and sinus symptoms, $19.24 \%$ of students had their symptoms for less than six weeks while $22.06 \%$ had their symptoms for more than six weeks but for less than a year.

The strong correlations between nasal and paranasal scores and sleep, social and emotional scores indicates the impact of nasal disease on quality of life including social limitation, time off school and reduced work productivity ${ }^{(7-11,20,21)}$. Subgroup analysis also showed correlations of cough symptoms with severe sinus or nasal scores, unlike adults ${ }^{(3)}$. Cough in children may be misdiagnosed as asthma.

Genetic involvement in disease expression is also confirmed by statistically significant worse scores of the MSYPQ in those with a family history of asthma, eczema, hay fever and food allergy. These findings are consistent both with the known family linkage of atopic disease and the association between asthma, rhinitis and eczema.

Treatment is from their G.P., over the counter, or alternative medicine. The cost is likely to be a significant burden to NHS and to the parents when the GP is not involved. Any cost of investigations is not included in this assessment.

In a survey of 70 students, the analysis of ARIA based questionnaire shows that over $37 \%$ have nasal symptoms for more than 4 days a week and more than 4 weeks at a time. This also confirms what the MSYPQ suggests: that a significant number of young people have persistent rhinitis. The burden of this problem is considerably under appreciated.

This research identifies that upper respiratory tract symptoms are highly prevalent within the 11-16 year old community and that there is considerable co-morbidity associated with this problem. Rhinitis/rhinosinusitis has not achieved the attention accorded to asthma; this study clearly demonstrates that this is a situation which needs to be rectified.

\section{Acknowlegdement}

The authors would like to acknowledge all the secondary school students who took part and the teachers involved; without their co-operation this would not have been possible. 


\section{Authorship contribution}

This work forms part of a submission for an MSc in Allergy. Dr

\section{Conflicts of Interest}

No conflict of interest exists.

Amtul Salam Sami is the researcher and author, Dr Glenis K Scad-

ding is the research supervisor and co-author.

\section{References}

1. Ghouri N, Hippisley-Cox J, Newton J, Sheikh A. Trends in the epidemiology and prescribing of medication for allergic rhinitis in England. J R Soc Med. 2008; 101: 466-472.

2. Lanza DC, Kennedy DW. Adult rhinosinusitis defined. Otolaryngol Head Neck Surg. 1997; 117: S1-S7.

3. Sami A. Epidemiology of Rhinitis in secondary school children using MSYPQ and comparison with Modified SNOT-20 used in adult community based survey. 2010 Nov 4; European Academy of Allergy and Clinical Immunology; 2010 p. 25.

4. Nunnaly JC. Psychometric theory. 2nd ed. New York: McGraw-Hill; 1978.

5. Walker S. Hay fever treatment-the essential skills. The Generalist. 2004; 2 (49)

6. Sibbald B, Rink E. Epidemiology of seasonal and perennial rhinitis: clinical presentation and medical history. Thorax. 1991; 46: 895901.

7. Bousquet J, Bullinger M, Fayol C, Marquis $P_{\text {, }}$ Valentin B, Burtin B. Assessment of quality of life in patients with perennial allergic rhinitis with the French version of the SF-36 Health Status Questionnaire. J Allergy Clin Immunol. 1994; 94: 182-188.

8. Simons FE. Learning impairment and allergic rhinitis. Allergy Asthma Proc. 1996; 17: 185-189.

9. Spaeth J, Klimek L, Mosges R. Sedation in allergic rhinitis is caused by the condition and not by antihistamine treatment. Allergy.
1996; 51: 893-906.

10. Vuurman EF, van Veggel LM, Uiterwijk MM Leutner D, O'Hanlon JF. Seasonal allergic rhinitis and antihistamine effects on children's learning. Ann Allergy. 1993; 71: 121-126.

11. Malone DC, Lawson KA, Smith DH, Arrighi HM, Battista C. A cost of illness study of allergic rhinitis in the United States. J Allergy Clin Immunol. 1997: 99: 22-27.

12. Ray NF, Baraniuk JN, Thamer M, Rinehart CS Gergen PJ, Kaliner M, et al. Healthcare expenditures for sinusitis in 1996: contributions of asthma, rhinitis, and other airway disorders. J Allergy Clin Immunol. 1999; 103: 408-414.

13. Guyatt GH, Cook DJ. Health status, quality of life, and the individual. JAMA. 1994; 272 630-631.

14. Torrance GW. Utility approach to measuring health-related quality of life. J Chronic Dis. 1987; 40: 593-603.

15. Tugwell P, Bombardier C, Buchanan WW Goldsmith CH, Grace E, Hanna B. The MACTAR Patient Preference Disability Questionnaire--an individualized functional priority approach for assessing improvement in physical disability in clinical trials in rheumatoid arthritis. J Rheumatol. 1987; 14: 446-451.

16. Jessen M, Malm L. Definition, prevalence and development of nasal obstruction. Allergy 1997; 52: 3-6.

17. Kirshner B, Guyatt G. A methodological framework for assessing health indices. J Chronic Dis. 1985; 38: 27-36.
18. Piccirillo JF, Edward D, Haiduck A Psychometric and clinimetric validity of the 31 items Rhinosinusitis outcome measure (RSOM-31). Am J Rhinol. 1995; 9: 297-306.

19. Piccirillo JF, Merritt MG, Jr., Richards ML. Psychometric and clinimetric validity of the 20-Item Sino-Nasal Outcome Test (SNOT20). Otolaryngol Head Neck Surg. 2002; 126 41-47.

20. Cockburn IM, Bailit HL, Berndt ER, Finkelstein SN. Loss of work productivity due to illness and medical treatment. J Occup Environ Med. 1999; 41: 948-953.

21. Gregory C, Cifaldi M, Tanner LA. Targeted intervention programs: creating a customized practice model to improve the treatment of allergic rhinitis in a managed care population. Am J Manag Care. 1999; 5: 485496.

\section{Dr Amtul Salam Sami}

1 Cow Leaze

Beckton

London, E6 6WX

United Kingdom

Tel: +44-20-7474 2595

Fax: +44-20-7474 7878

Email:amtul_salam@hotmail.com 